\title{
Addendum: The muscle-specific microRNA miR-1 regulates cardiac arrhythmogenic potential by targeting GJA1 and KCNJ2
}

Baofeng Yang, Huixian Lin, Jiening Xiao, Yanjie Lu, Xiaobin Luo, Baoxin Li, Ying Zhang, Chaoqian Xu, Yunlong Bai, Huizhen Wang, Guohao Chen \& Zhiguo Wang

Nat. Med. 13, 486-497 (2007); published online 1 April 2007; addendum published after print 6 December 2011

Editors' note: With regard to the above article, the editors wish to notify readers of Nature Medicine that the Montreal Heart Institute announced on 2 September 2011 that an investigation had found evidence of misconduct in publications from the laboratory of Zhiguo Wang at that institution. The committee in charge of this investigation recommended that several publications from Z. Wang's laboratory be retracted (some of which had already been retracted over the summer); in addition, his laboratory at the Montreal Heart Institute was closed. One of the papers investigated was the Nature Medicine paper listed above, for which the corresponding authors were Z. Wang (affiliated with the Harbin Medical University, China and the Montreal Heart Institute, Canada) and Baofeng Yang (affiliated with the State-Province Key Laboratories of Biomedicine-Pharmaceutics of China and the Institute of Cardiovascular Research, Harbin Medical University, China). The investigating committee's report stated that they did not find evidence of misconduct for the data in the Nature Medicine paper that was generated at the Montreal Heart Institute; namely, Figure la ("CAD human" data only) and Figure 2f,g. According to the report, the remaining data in the paper were not generated at the Montreal Heart Institute and were not investigated. In correspondence with Nature Medicine, both Z. Wang and Yang stand by the data in the Nature Medicine paper. However, Z. Wang noted that, for Figure 2b, lanes from the original blot had been rearranged in the published figure, for which we are publishing a Corrigendum in this issue.

\section{Corrigendum: The muscle-specific microRNA miR-1 regulates cardiac arrhythmogenic potential by targeting GJA1 and KCNJ2}

\begin{abstract}
Baofeng Yang, Huixian Lin, Jiening Xiao, Yanjie Lu, Xiaobin Luo, Baoxin Li, Ying Zhang, Chaoqian Xu, Yunlong Bai, Huizhen Wang, Guohao Chen \& Zhiguo Wang

Nat. Med. 13, 486-497 (2007); published online 1 April 2007; corrected after print 6 December 2011

In the version of this article initially published, lanes from the original blot shown in Figure $2 \mathrm{~b}$ (NIZ and IZ samples, blotting for 55-kDa Kir2.1 and GAPDH) were rearranged in the published figure. The two lanes at the far right of the published blot were on the far left of the original blot, so that the sequence of the lanes in the original blot was as follows (from left to right): IZ (WT miR-1 + AMO-1), IZ (MT miR-1), NIZ, IZ, IZ (AMO-1), IZ (WT miR-1). We have added white space to indicate that the blot is not continuous in the HTML and PDF versions of the article.
\end{abstract}

\section{Corrigendum: Targeting osteoclast-osteoblast communication}

$\mathrm{Xu}$ Cao

Nat. Med. 17, 1344-1346 (2011); published online 7 November 2011; corrected after print 7 November 2011

In the version of this article initially published in print, the figure had incorrectly shown the receptor on the osteoclast progenitor as RANKL and the corresponding ligand on the osteoblast as RANK, instead of RANK on the osteoclast progenitor and RANKL on the osteoblast. The figure labeling was always correct in the HTML and PDF versions of the article.

\section{Corrigendum: Resolving controversies on the path to Alzheimer's therapeutics}

Dennis J Selkoe

Nat. Med. 17, 1060-1065 (2011); published online 7 September 2011; corrected after print 21 October 2011

In the version of this article initially published, the name of the antibody bapineuzumab was incorrectly spelled as 'bapineuzimab' throughout the text. The error has been corrected in the HTML and PDF versions of the article. 